\title{
Orbital apex syndrome caused by aspergilloma in an immunocompromised patient with cutaneous lymphoma: A case report of a rare entity
}

\author{
${ }^{1}$ Department of Neurosurgery, Sana Hospitals Duisburg, Duisburg, Germany \\ ${ }^{2}$ Institute of Neuropathology, University of Duisburg-Essen, Essen, Germany \\ ${ }^{3}$ Neurosurgical Division, Department of Surgery, Lagos University Teaching Hospital, Lagos, Nigeria
}

A Cheko, ${ }^{1}$ MDS; S Jung, ${ }^{1}$ MD; S Teuber-Hanselmann, ${ }^{2} \mathrm{MD} ;$ A W Oseni, ${ }^{3} \mathrm{MD}, \mathrm{MSc} ; \mathbf{A}$ Tsogkas, ${ }^{1} \mathrm{MD} ; \mathbf{M}$ Scholz,${ }^{1} \mathrm{MD} ;$ A K Petridis, ${ }^{1} \mathrm{MD}$

Corresponding author: A W Oseni (oseniabidemi@yahoo.com)

A 57-year-old man with a history of chemotherapy because of cutaneous lymphoma presented with an orbital apex syndrome. The cranial computed tomography scan revealed a tumour in the orbital apex, extending intradurally. With a suspected diagnosis of a neoplastic lesion, the patient underwent orbital surgery with optic nerve decompression. Histology revealed an aspergilloma. No other foci were seen and treatment with antifungals was started. In immunocompromised patients with intracranial tumours, infection is always a major consideration in the differential diagnosis, even if the reason for immunosuppression (in this case chemotherapy) dates back several months. Misdiagnosing an orbital apex lesion as a cancer and treating patients primarily with corticosteroids can be life threatening. Removal or biopsy of such lesions is essential in further treatment since antifungals have to be administered as fast as possible.

S Afr Med J 2016;106(4):354-355. DOI:10.7196/SAMJ.2016v106i4.9936

Aspergillosis of the brain is very rare, and usually seen in immunocompromised or immunosuppressed patients. ${ }^{[1]}$ Cases where aspergillomas affect the orbital apex are more rare and a literature search offers only 13 search results. Orbital aspergillomas are associated with infections of the paranasal sinuses. The spores are commonly airborne and inhaled by the host, causing infection of the respiratory system. ${ }^{[2,3]}$ Aspergillus flavus may gain access to the central nervous system (CNS) via haematogenous spread or direct spread from the paranasal sinuses. ${ }^{[2]}$ The ethmoidal and sphenoidal sinuses are usually the loci of the infection, which in immunocompromised individuals, runs a fulminant course unlike the insidious onset in healthy patients. ${ }^{[4]}$ Invasive aspergillosis is a disease of concern, as it is a leading cause of death in patients with haematological malignancies, those who are immunocompromised, and transplant recipients. ${ }^{[5]}$

Diagnosis requires a high index of suspicion and needs special treatment with mandatory biopsy or the surgical removal of lesions close to the orbit, especially in immunocompromised patients. Antifungal therapy should start as soon as possible after diagnosis. We present a case of a patient treated with chemotherapy for a cutaneous lymphoma who developed an orbital apex syndrome 6 months after his last chemotherapy cycle.

\section{Case report}

A 57-year-old patient with features suggestive of an orbital apex syndrome for approximately 5 weeks presented to our department. The main complaints were double vision, opthalmoplegia and visual disturbance. Two days prior to admission he lost vision in the left eye. A cranial computed tomography (CT) scan was performed and a mass around the left orbital apex invading the superior orbital fissure and the optic canal with intradural growth could be seen (Fig. 1). A diagnosis of metastasis was entertained in view of the patient's background history of cutaneous B-cell lymphoma. No signs of paranasal sinus infection were seen.

Corticosteroid therapy had been initiated and the patient was scheduled for tumour removal and optical nerve decompression. The mass was completely excised, and the immediate postoperative period was uneventful. However, histology of the excised mass revealed it to be an aspergilloma. Corticosteroids were ceased and intravenous antifungal therapy was immediately initiated. The patient recovered rapidly, particularly his visual problems. Further investigations did not reveal other lesions. Paranasal sinuses, lungs and abdomen were free of any suspicious findings.

\section{Discussion}

Aspergillomas of the orbit were reported more often in the 1960s and 1970s and less in the 1980s and 1990s. There was an increase

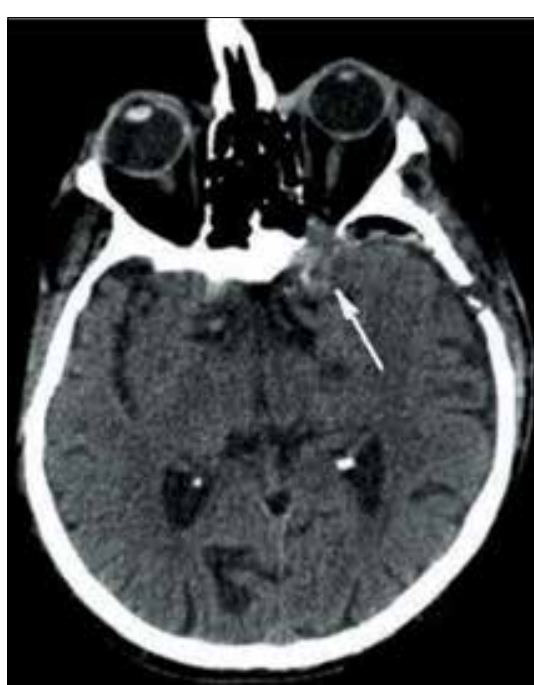

Fig. 1. Cranial CT showing a tumourous mass in the left orbital apex (white arrow).

in reports in the first decade of the 20th century. With more efficient antimycotic agents, the incidence of mycotic orbital infections decreased between 1980 and 2000. There was an increase in incidence from 2000 to 2010 since aggressive chemotherapeutic regimens and HIV led to seriously immunocompromised patients. ${ }^{[6]}$ However, diagnostic imaging became much more efficient and the lesions could be more easily seen. Since we now have the diagnostic tools to identify such lesions and an increased report rate, the way to avoid fatal misdiagnosis and incorrect treatment 


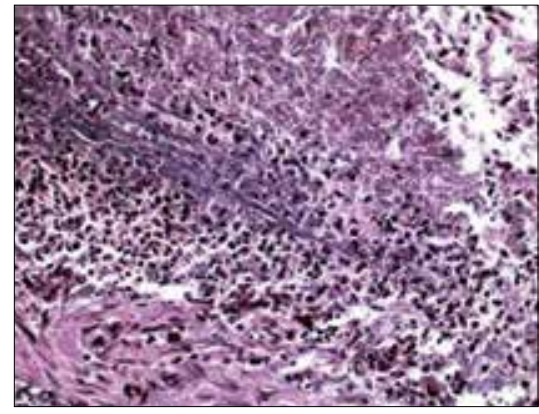

Fig. 2. Connective tissue with dense mononuclear infiltrate predominantly composed of neutrophil granulocytes and adjacent cellular detritus (haematoxylin eosin staining, 20-fold image magnification).

strategies is to consider these entities during our differential diagnostic algorithm.

In a number of cases, infections began in one of the near sinuses and they could be granulomatous or non-granulomatous. ${ }^{[7]}$ The granulomatous entities can cause a proptosis as well as other clinical signs, whereas the nongranulomatous may be asymptomatic. ${ }^{[3,8]}$ More importantly, even histologically the fungus granuloma of the orbit could be misdiagnosed as a pseudotumour when not specially stained. ${ }^{[3]}$ The granulomatous appearance on haematoxylin eosin staining is that of connective tissue with dense mononuclear infiltrate (Fig. 2). Treating such a patient for a tumour could allow the fungus to grow and

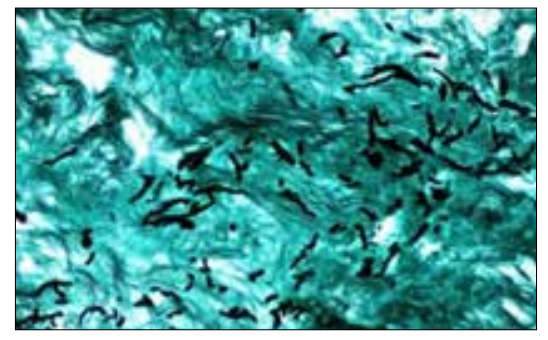

Fig. 3. Grocott staining reveals septated fungal hyphae, partially showing branching with an angle of $45^{\circ}$ as it is seen in Aspergillus spp. (20fold image magnification).

prove fatal. In a very honest case report from 1970, the authors reported a case of a 40-yearold female patient with a proptosis who was diagnosed as having a pseudotumour. ${ }^{[7]}$ Steroid and antimicrobial therapy did not improve the patient's condition. The authors proceeded with an orbitotomy and suspected a tuberculoma. However, after the treatment with antituberculotics failed to have any effect, they reviewed and scrutinised the histological specimen; on Gridley's stain they diagnosed an aspergilloma. They could not treat the patient with antimycotics, however, because she was lost to follow-up. ${ }^{[7]}$

The authors had only X-ray imaging in their armamentarium and did not give up on questioning their own diagnosis since in close follow-up, no effects of their treatment could be seen. What led to the right diagnosis was persistent follow-up, doubting their diagnosis and correcting it and the permanent suspicion of another agent than the usual ones. In addition they were brave enough to share their mistakes and publish. We should feel obliged to share our experiences with such rare diseases, to avoid mistreatment, and to make the right diagnosis with specific staining patterns (Fig. 3), and to choose the right therapies.

High index of suspicion of possibility of fungal infection in immunocompromised patients, avoidance of steriods coupled with early surgical decompression and commencement of antifungal treatment leads to an improved outcome.

1. Chowdhury FH, Haque MR, Khan SK, Alam SM. Cerebral aspergilloma in a SLE patient: A case report with short literature review.

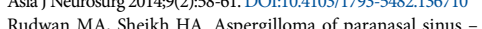
2. Rudwan MA, Sheikh HA. Aspergilloma of paranasal sinus - a common cause of unilateral proptosis in Sudan. Clin Radio 3. Barr A, Nolan M, Grant W, Costello C, Petrou MA. Rhinoorbital and . Barr A, Nolan M, Grant W, Costello C, Petrou MA. Rhinoorbital and
pulmonary zygomycosis post pulmonary aspergilloma in a patient with chroniclymphocyticleukemia. Acta Biomed 2006;77(Suppl 4):13-18. 4. Naik MN, Vemuganti GK, Honavar SG. Primary orbital aspergilloma of the exenterated orbit in an immunocompromized patient. Indian J Med Microbiol 2006;24(3):233-234.

5. Segal BH. Aspergillosis. N Engl J Med 2009;360(18):1870-1884. DOI:10.1056/NEJMra0808853

6. Busch M, Weiler H, Frühmorgen P, Rühl U, Roos W. Paranasal sinus mycetoma with orbital involvement in a patient with AIDS. Bildgebung 1995;62(3):199-201.

7. Chandra P, Ahluwalia BK, Chugh TD. Primary orbital aspergilloma. Br J Ophthalmol 1970;54:693-696.

8. Goel A, Nadkarni T, Desai AP. Aspergilloma in the Goel A, Nadkarni T, Desai AP. Aspergilloma in the
paracavenous region - two case reports. Neurol Med Chir (Tokyo) 1996;36(10):733-736. DOI:10.2176/nmc.36.7330

Accepted 3 October 2015 